Original Article

\title{
Salvianolic acid B ameliorates psoriatic changes in imiquimod- induced psoriasis on BALB/c mice by inhibiting inflammatory and keratin markers via altering phosphatidylinositol-3-kinase/ protein kinase B signaling pathway
}

\author{
Shoufan Wang, Lihong Zhu, Yihou Xu, Zongbi Qin, and Aiqin Xu* \\ Department of Dermatology, Wuhan Hospital of Traditional Chinese Medicine, Wuhan, Hubei 430000, China
}

\section{ARTICLE INFO}

Received September 18, 2019

Revised January 12, 2020

Accepted January 14,2020

*Correspondence

Aiqin Xu

E-mail:39260467@qq.com

\section{Key Words}

Inflammatory markers

Keratin markers

PASI

PI3K/Akt signaling pathway

Salvianolic acid B
ABSTRACT Salvianolic acid B (SAB) is an active phytocomponent of a popular Chinese herb called Radix Salvia militiorrhiza with numerous biological properties. The anti-psoriasis activity of SAB was examined by evaluating various psoriasis inflammatory and keratin markers against imiquimod (IMQ)-induced psoriasis on BALB/c mice. Totally 50 healthy BALB/c mice were evenly divided into 5 groups including control, drug control (SAB; $40 \mathrm{mg} / \mathrm{kg}$ ), IMQ-induced psoriasis (5\%), IMQ exposure and treated with $\mathrm{SAB}(40 \mathrm{mg} / \mathrm{kg})$, or standard methotrexate (MTX; $1 \mathrm{mg} / \mathrm{kg})$. Mice supplemented with either SAB or MTX significantly lowered the values of psoriasis area severity index (PASI), erythema, scaling, skin thickness, inflammatory markers (interleukin [IL]$22 / 23 / 17 A / 1 \beta / 6$ ) and lipid peroxidation product (malondialdehyde). Also, IMQ exposed BALB/c mice treated with SAB or MTX display lesser histopathological changes with enhanced antioxidant activities (catalase, superoxide dismutase). Moreover, the protein expression of keratin markers (K16 and K17) and phosphatidylinositol3-kinase ( $\mathrm{PI} 3 \mathrm{~K}) /$ protein kinase B (Akt) signaling proteins (pAkt/Akt and pPI3K/PI3K) were significantly downregulated after administration with $S A B$ and MTX as compared with IMQ induced mice. Taking together, SAB and MTX significantly ameliorate psoriatic changes by inhibiting psoriatic inflammatory and keratin markers through abolishing PI3K/Akt signaling pathway. However, further studies (clinical trials) are needed to confirm the anti-psoriatic property of SAB before recommending to psoriasis patients.

\section{INTRODUCTION}

Psoriasis is a common chronic T-cell mediated autoimmune dermal disease, which affects approximately $2 \%-3 \%$ of the global population [1]. Reports have shown that psoriasis mostly affects the skin at the head (scalp), arms, elbow, knees, and shoulder [2]. Hence most of the psoriatic patients experience social discrimination and stigma (mental stress) due to their appearance and thus pose a huge negative impact on the quality of life [3]. The major dermal pathological events in psoriasis include hyperproliferation of epidermis (acanthosis) abnormal or hyper-differentiation of keratinocytes (parakeratosis) or plasmacytoid dendritic cells, excess infiltration of leukocytes (neutrophils) along with enhanced pro-inflammatory cytokines production and angiogenesis $[4,5]$. In particular, Th17 (T helper cells) is directly involved in the production of various inflammatory cytokines (interleukin [IL]-17 and 23) and has a direct influence on psoriasis [6]. At present only a few therapeutic strategies are used for treating or managing pso- (i) \$ This is an Open Access article distributed under the terms of the Creative Commons Attribution Non-Commercial License, which permits unrestricted non-commercial use, distribution, and reproduction in any medium, provided the original work is properly cited. Copyright @ Korean J Physiol Pharmacol, pISSN 1226-4512, eISSN 2093-3827
Author contributions: S.W., A.X., and L.Z. involved in conception and designing this study. Y.X. and Z.Q. conducted experiment and acquisition of data. S.W., L.Z., and A.X. analysis and interpretation of data. S.W., Z.Q., and A.X. drafted and revised the manuscript. 
riasis which includes anti-inflammatory agents like ustekinumab (mAb against interleukins especially 23A and 12), tumor necrosis factor (TNF)- $\alpha$ inhibitor and immunosuppressants like corticosteroids. Also, few drugs are under pipeline with promising results in phase II and III clinical trials $[7,8]$. Nevertheless, those anti-psoriatic treatments might result in serious adverse effects like nasopharyngitis, respiratory infections as well as expensive and which trigger many scientists to focus on developing much safer and less expensive natural drug (alternative/complementary medicine) with potent immunomodulatory and anti-inflammatory activities to combat against psoriatic changes and its related complications $[9,10]$.

Radix Salvia militiorrhiza (Danshen/red sage; dried root) is a popular Chinese herb, which is recommended for treating dermal disorders, cardiovascular and cerebrovascular diseases as well as rheumatoid arthritis and chronic pain [11]. Radix Salvia militiorrhiza dried root has several biologically active phytocomponents, out of which salvianolic acid B (SAB) is one of the abundant active phytocomponent with many beneficial properties like antiinflammatory, antioxidant, anti-tumor/cancer, anti-diabetic [12-14]. Also, SAB is beneficial against various auto-immune disorders including rheumatoid arthritis and alopecia owing to its potent immunomodulatory and anti-inflammatory activities [15]. In addition, salvianolic acid B was reported to suppress melanin proliferation (production) and thus exhibit its derma protective property [16]. Based on the above reports, we hypothesize that salvianolic acid (potent immunomodulatory, anti-inflammatory and derma protective properties) would abolish psoriatic changes by lowering inflammatory markers in imiquimod (IMQ)-induced psoriasis.

\section{METHODS}

\section{Chemicals and drugs}

SAB (98\% pure), sodium dodecyl sulfate (SDS), paraformaldehyde, glycerol, and bromophenol blue were bought from Sigma Aldrich (St. Louis, MO, USA). Topical IMQ (5\%) and methotrexate (MTX) were purchased from Sichuan Mingxin Pharmaceuticals Co., Ltd. (Sichuan, China). All the other chemicals and reagents used in this study are of either analytical or HPLC grade.

\section{Experimental animal and ethical approval}

Healthy BALB/c mice of male gender aged between 7 to 8 weeks, weighing $23 \pm 5 \mathrm{~g}$ were procured from Suzhou Fengshi Laboratory Animal Equipment and Merchant Co., Ltd. (Suzhou, China). All the mice were maintained at optimal laboratory conditions $\left(22^{\circ} \mathrm{C} \pm 2{ }^{\circ} \mathrm{C} ; 55 \%-60 \%\right.$ humidity) in the polycarbonate cage. Mice have full access to mice fed and water (ad libitum) under $12 \mathrm{~h}$ light/dark cycle every day. All the mice were shaved at the back $(3 \times 4 \mathrm{~cm})$ and allow for a week as an assimilation period. All the animal procedures and protocols were approved by the institutional animal ethical board of Wuhan Hospital of Traditional Chinese Medicine (No. WH/12a-34-2018). Mice were handled with extreme care based on guidelines put forth by National Institutes of Health for Care and Use of laboratory animals.

\section{Induction of psoriasis}

Psoriasis in $\mathrm{BALB} / \mathrm{c}$ mice was induced by topical application of IMQ (5\%; $62.5 \mathrm{mg}$ ) on the shaved back of all mice (except control and $\mathrm{SAB}$ alone group mice) for 7 days as previously mentioned by El Malki and his co-workers [17].

\section{Experimental animals grouping}

Totally 50 healthy male BALB/c mice were divided into 5 groups with 10 in each group. Control group mice $(\mathrm{n}=10)$ received the only saline for 7 days, drug control group mice received only SAB (40 mg/kg via i.p. mixing with saline) for all for 7 days. Whereas, IMQ-induced psoriasis model mice were topically applied with 5\% IMQ as indicated in the above section (induction of psoriasis) for 7 continuous days. The treatment group was pre-treated with either SAB (40 mg/kg) or MTX (1 mg/kg) for 7 days and followed by 7 days of IMQ induction as well as cotreatment with SAB or MTX. Overall, 7 days of pre and 7 days of co-treatment with SAB or MTX. But SAB alone and control mice received only 7 days of either saline or $S A B$.

\section{Sample collection and processing}

After 7 days of IMQ induction, all mice were fasted (overnight) and on 8th day morning all mice were weighed and euthanized under strong sodium pentobarbital at a dose of $55 \mathrm{mg} / \mathrm{kg}$ and the shaved area (back) were removed (epidermis) softly and gently as well as spleen were collected immediately and dry weight were weighed (to check spleen index: spleen weight/body weight). The dermal sample was segregated into two portions, one for biochemical/molecular analysis and other portion for histopathological analysis (fixed in 10\% paraformaldehyde). The dermal tissue was homogenized with phosphate saline buffer solution ( $\mathrm{pH}$ 7.4) and centrifuged $\left(15,000 \mathrm{~g}\right.$ at $4^{\circ} \mathrm{C}$ for $\left.10 \mathrm{~min}\right)$ and the supernatant was used for biochemical and molecular analysis. The psoriasis area severity index (PASI) score (0 to 4 ) including the skin erythema and scaling were measured (lesioned area) to monitor the grade of psoriasis severity on the $2 \mathrm{nd}, 4 \mathrm{th}$, 6th, and 8 th day based on scoring range from 0 to 4 . Where 0 represents none, 1 represents slight, 2 represents moderate, 3 represents marked/high and 4 represents very marked/very high as mentioned previously by Zhang et al. [18]. Moreover, the skin (dermal) thickness was measured on the 8th day (before sacrifice) using thickness gauge/ caliper bought from Guanglu Digital Caliper Manufacturer Co., 
Ltd. (Beijing, China).

\section{Lipid peroxidation products and antioxidants}

The skin lipid peroxidation product (malondialdehyde, MDA) and antioxidant activities (catalase, CAT; superoxide dismutase, SOD) were determined using commercial assay kit bought from Nanjing Bio-engineering Institute, Co. (Nanjing, China) based on suppliers' protocol.

\section{Inflammatory markers/mediators}

The concentration of various inflammatory markers like IL$23 / 22 / 17 \mathrm{~A} / 1 \beta$ and TNF- $\alpha$ in dermal tissue homogenate were measured using commercial ELISA kit purchased from R \& D Systems (Minneapolis, MN, USA) in accordance with manufacturers instruction.

\section{Western blot}

The dermal tissue homogenate was incubated with RIPA lytic buffered solution containing various proteases and centrifuged and the resultant supernatant was used to estimate the protein level using Pierce BCA protein assay kit (Abcam, Cambridge, UK). An equal amount of protein sample (each group) was resolved and separated by $8 \%$ (keratin) and 10\% (PI3k and Akt) SDS-PAGE apparatus and electro-transferred onto polyvinylidene difluoride (PVDF) membrane. The PVDF membrane was washed with PBS and followed by treatment with non-fat milk (5\%), Tween 20, PBS. Then the membrane was probed with primary antibodies (bought from Cell Signaling Technology, Danvers, MA, USA) such as rabbit monoclonal anti-K16 $(1: 1,000)$, anti-K17 (1:1,200), rabbit polyclonal anti-pPI3K110 and PI3K (1:1,000), rabbit monoclonal anti-pAkt $\mathrm{Ser}^{473}$ and Akt $(1: 1,000)$ as well as housekeeping protein mouse polyclonal GADPH $(1: 1,500)$ for overnight at $4^{\circ} \mathrm{C}$. Then the membrane was washed twice with PBS to remove unbound antibodies. Finally, the PVDF membrane was again incubated with secondary antibody-anti-horseradish peroxidase (HRP) anti-donkey IgG antibody $(1: 10,000)$ for $1 \mathrm{~h}$ at $37^{\circ} \mathrm{C}$. The protein intensity (band) in the PVDF membrane was developed using Enhanced Chemiluminescence (ECL) detection system and the picture of the protein bands were quantified using Image Lab software; (V6.0) from Bio Rad Laboratories, Inc. (Hercules, CA, USA).

\section{Histopathological analysis}

As mentioned in the sample section, a portion of dermal tissue was fixed in $10 \%$ paraformaldehyde and treated with graded series of ethyl alcohol (rehydrate) and embedded with liquid paraffin wax to make a solid dermal tissue block. Tissue block was sectioned into a thin slice $(4 \mu \mathrm{m})$ using ultra-microtome and mixed with xylene and mounted on a microscopic slide and stained with hematoxylin and eosin (H\&E) stain for $12 \mathrm{~h}$ at room temperature. Then, the stained tissue slides were visualized using a light microscope at a magnification of 400 $\times$ and pictured (BX43; Olympus Co., Tokyo, Japan) to examine any skin morphological changes with the help of expert pathologist who is blinded from grouping or experiment.

\section{Data analysis}

For the present study, the data analysis was conducted using Statistical Package for the Social Software-SPSS (ver 21; IBM, Armonk, NY, USA). Values are expressed as the mean \pm standard deviation. The statistical difference (p-value) between the experimental groups was assessed by one-way ANOVA followed by Duncan's multi-comparison test. $\mathrm{p}<0.05$ is deemed statistically significant and were expressed with different symbols.

\section{RESULTS}

\section{Impact of SAB/MTX on spleen index}

The spleen index was calculated by dividing spleen weight (dry weight)/body weight to assess the dynamic body changes. Both control (0.005) and drug control (0.005) mice showed a lesser spleen index. Whereas, IMQ exposed mice (0.012) displayed higher spleen index $(\mathrm{p}<0.01)$ than control mice. Nevertheless, IMQ-induced mice pre-treated and co-treated with SAB (0.008) and MTX $(0.008)$ significantly reduced $(\mathrm{p}<0.01)$ the spleen index than IMQ-exposed BALB/c mice.

\section{Impact of SAB/MTX on PASI, erythema, scaling and skin thickness}

Fig. 1 showcase the impact of SAB on cumulative PASI (scaling/ erythema), dermal scaling and erythema as well as skin thickness (dorsal skin) in control and IMQ-induced mice. Topical application of IMQ (5\%) resulted in elevated psoriatic plaque, erythema, scaling and inflamed dermal lesion, which are reflected in a significant increase $(\mathrm{p}<0.01)$ in the levels of PASI (Fig. 1A), scaling (Fig. 1B), erythema (Fig. 1C) scores and skin thickness (Fig. 1D) than control mice. Whereas, 7 days of pre and co-treatment with SAB or MTX showed a significant decrease $(\mathrm{p}<0.01)$ in the levels of PASI, scaling, erythema scores and skin thickness as compared to IMQ-induced mice. Both the control and SAB alone (drug control) mice did not show any psoriatic changes (symptoms) with normal skin texture. 
A

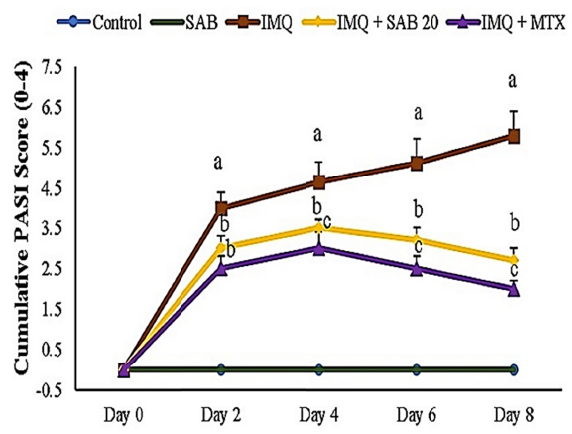

C
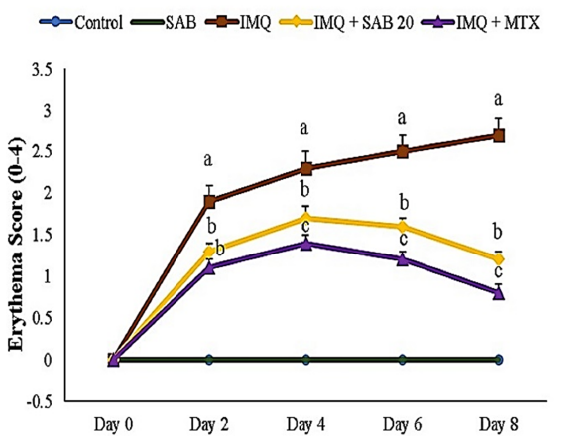

B

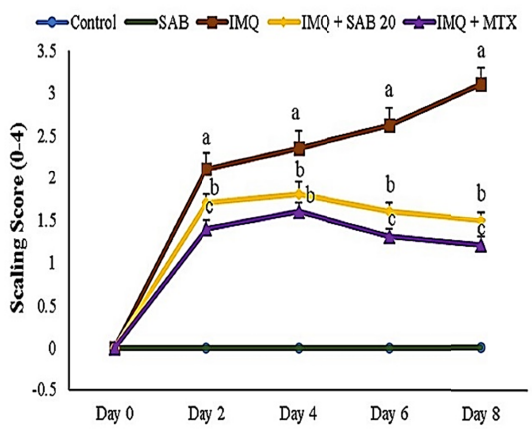

D

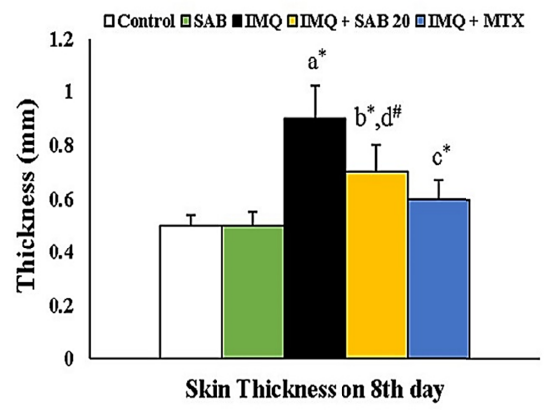

Fig. 1. Illustrate the skin PASI (A), scaling (B), erythema (C) and thickness (D) (only on 8th day) in control and IMQ-induced mice. Values are expressed as the mean \pm standard deviation. IMQ, imiquimod; SAB, salvianolic acid B; MTX, methotrexate; PASI, psoriasis area severity index. $p$-value ( $\mathrm{p} p$ $<0.05$, ${ }^{*} p<0.01$ ): where 'a' denotes the comparison between IMQ vs. Control, ' $b$ ' denotes the comparison between IMQ + SAB vs. IMQ, 'c' denotes the comparison between IMQ + MTX vs. IMQ, 'd' denotes the comparison between IMQ + SAB vs. IMQ + MTX.

Table 1. Exemplifies the skin lipid peroxidation products and antioxidant activities in control and IMQ-induced mice

\begin{tabular}{|c|c|c|c|}
\hline Parameters & MDA (nmol/mg protein) & CAT (U/mg protein) & $\mathrm{SOD}(\mathrm{U} / \mathrm{mg}$ protein) \\
\hline Control & $10.64 \pm 1.80$ & $21.50 \pm 2.40$ & $39.22 \pm 4.50$ \\
\hline $\mathrm{SAB}$ & $11.02 \pm 1.75$ & $21.00 \pm 2.25$ & $38.85 \pm 4.09$ \\
\hline $\mathrm{IMQ}$ & $28.95 \pm 3.70^{\mathrm{a} *}$ & $09.88 \pm 1.10^{a *}$ & $20.40 \pm 2.20^{\mathrm{a} *}$ \\
\hline $\mathrm{IMQ}+\mathrm{SAB}$ & $18.61 \pm 2.35^{\mathrm{b}_{*}, \mathrm{~d} \#}$ & $17.10 \pm 3.00^{b_{*}, \mathrm{~d} *}$ & $33.68 \pm 3.80^{\mathrm{b} *, \mathrm{~d} *}$ \\
\hline $\mathrm{IMQ}+\mathrm{MTX}$ & $20.05 \pm 2.03^{\mathrm{c} *}$ & $14.75 \pm 2.05^{\mathrm{c} \sharp}$ & $29.79 \pm 3.15^{c *}$ \\
\hline
\end{tabular}

Values are expressed as the mean \pm standard deviation. IMQ, imiquimod; MDA, malondialdehyde; CAT, catalase; SOD, superoxide dismutase; SAB, salvianolic acid B; MTX, methotrexate. $p$-value $\left({ }^{*} p<0.05,{ }^{*} p<0.01\right)$ : where ' $a$ ' denotes the comparison between IMQ vs. Control, ' $b$ ' denotes the comparison between IMQ + SAB vs. IMQ, 'c' denotes the comparison between IMQ + MTX vs. IMQ, 'd' denotes the comparison between IMQ + SAB vs. IMQ + MTX.

Table 2. Exemplifies the skin inflammatory markers in control and IMQ-induced mice

\begin{tabular}{lccccc}
\hline Parameters & IL-23 $(\mathrm{pg} / \mathrm{mg}$ protein) & IL-22 $(\mathrm{pg} / \mathrm{mg}$ protein) & IL-17A (pg/mg protein) & IL-1 $\beta$ (ng/mg protein) & TNF- $\alpha$ (ng/mg protein) \\
\hline Control & $15.80 \pm 1.75$ & $1.10 \pm 0.15$ & $7.55 \pm 0.75$ & $7.42 \pm 0.71$ & $5.10 \pm 0.50$ \\
SAB & $16.00 \pm 2.10$ & $1.05 \pm 0.10$ & $7.40 \pm 0.58$ & $7.45 \pm 0.80$ & $4.97 \pm 0.52$ \\
IMQ & $41.25 \pm 4.60^{\mathrm{a} *}$ & $3.87 \pm 0.40^{\mathrm{a} *}$ & $13.98 \pm 1.80^{\mathrm{a} *}$ & $25.10 \pm 2.45^{\mathrm{a} *}$ & $15.04 \pm 1.85^{\mathrm{a} *}$ \\
$\mathrm{IMQ}+\mathrm{SAB}$ & $23.50 \pm 3.00^{\mathrm{b} *, \mathrm{~d} *}$ & $2.02 \pm 0.20^{\mathrm{b} *, \mathrm{~d}, \mathrm{NS}}$ & $9.75 \pm 1.10^{\mathrm{b} *, \mathrm{~d}, \mathrm{NS}}$ & $11.21 \pm 1.35^{\mathrm{b} * \mathrm{~d} *}$ & $7.14 \pm 0.97^{\mathrm{b} *, \mathrm{~d} *}$ \\
$\mathrm{IMQ}+\mathrm{MTX}$ & $25.18 \pm 2.10^{\mathrm{c} *}$ & $2.22 \pm 0.22^{\mathrm{c} *}$ & $9.50 \pm 1.12^{\mathrm{c} *}$ & $13.76 \pm 1.50^{\mathrm{c} *}$ & $8.95 \pm 0.88^{\mathrm{c} *}$ \\
\hline
\end{tabular}

Values are expressed as the mean \pm standard deviation. IMQ, imiquimod; IL-23/22/17A/1 $\beta$, interleukins 23/22/17A/1 beta; TNF- $\alpha$, tumour necrosis factor alpha; SAB, salvianolic acid B; MTX, methotrexate; NS, non-significant. $p$-value $\left({ }^{*} p<0.05,{ }^{*} p<0.01\right)$ : where 'a' denotes the comparison between IMQ vs. Control, ' $b$ ' denotes the comparison between IMQ + SAB vs. IMQ, ' $C^{\prime}$ denotes the comparison between IMQ + MTX vs. IMQ, 'd' denotes the comparison between IMQ + SAB vs. IMQ + MTX. 


\section{Impact of SAB/MTX on lipid peroxidation products and antioxidants}

Table 1 exemplifies the skin lipid peroxidation products and antioxidant activities in control and IMQ-induced mice. An exponential increase in the levels of lipid peroxidation and substantial decrement in the activity of dermal antioxidants like CAT and SOD were observed in IMQ-induced mice ( $<<0.01)$. However, the mice injected with SAB or MTX (before and after IMQ-induction) showed a notable decrease in the levels of lipid peroxidation as well as a considerable increment $(\mathrm{p}<0.01)$ in the antioxidant's activity of CAT and SOD on equivalent with IMQinduced psoriatic mice. In the case of antioxidant status, the SAB exhibits potent antioxidant activity than MTX.

\section{Impact of SAB/MTX on inflammatory markers/ mediators}

As shown in Table 2, the concentration of various dermal inflammatory markers/mediators like IL-23, 22, 17A, $1 \beta$ and TNF- $\alpha$ were exponentially elevated in IMQ-exposed mice ( $\mathrm{p}<$ 0.01 ) in parallel to control mice. Nevertheless, those elevated skin inflammatory markers/ mediators like IL-23, 22, 17A, 1 $\beta$, and TNF- $\alpha$ were significantly $(\mathrm{p}<0.01)$ abolished after treatment with SAB or MTX (before and after IMQ induction). Also, on a

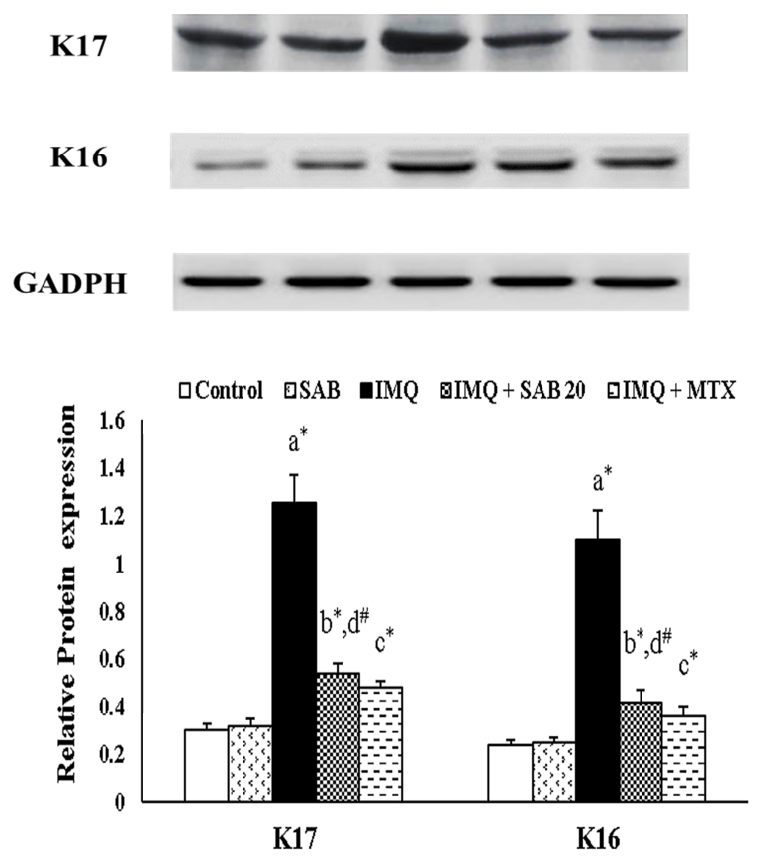

Fig. 2. Illustrate the protein expression of skin keratin markers (K16 and $\mathrm{K} 17$ ) in control and IMQ-induced mice. Values are expressed as the mean \pm standard deviation. IMQ, imiquimod; $S A B$, salvianolic acid B; MTX, methotrexate; K16 and K17, keratin 16/17. p-value ( ${ }^{*} \mathrm{p}<0.05$, ${ }^{*} \mathrm{p}<0.01$ ): where ' $a$ ' denotes the comparison between IMQ vs. Control, ' $b$ ' denotes the comparison between IMQ + SAB vs. IMQ, ' $c$ ' denotes the comparison between IMQ + MTX vs. IMQ, 'd' denotes the comparison between IMQ + SAB vs. IMQ + MTX. comparison between both treatment groups, the standard MTX showed slightly better anti-inflammatory activity than SAB.

\section{Impact of SAB/MTX on the protein expression of keratin markers and PI3K/Akt signaling molecules}

The protein expression of keratin markers (K16 and K17; Fig. 2) and PI3K/Akt signaling molecules (Fig. 3) were quantified using the western blot technique [19]. The mice topically applied with $5 \%$ IMQ $(\mathrm{p}<0.01)$ showed greater upregulation of keratin markers (K16, K17) and PI3K/Akt signaling molecules (pAkt/Akt and $\mathrm{pPI} 3 \mathrm{~K} / \mathrm{PI} 3 \mathrm{~K}$ ratio). Whereas, mice supplemented with SAB ( $\mathrm{p}<$ 0.01 ) or MTX ( $p<0.01)$, significantly downregulated the protein expression of keratin markers (K16, K17) and PI3K/Akt signaling molecules (pAkt/Akt and pPI3K/PI3K ratio) as compared to IMQ-exposed mice and thus exhibiting potent anti-psoriasis activity. Nonetheless, MTX showed superior anti-psoriasis activity than SAB due to its strong immunomodulatory property.

\section{Impact of SAB/MTX on histopathological changes in dermal tissue}

Fig. 4 illustrates the histopathological changes in dermal tissue

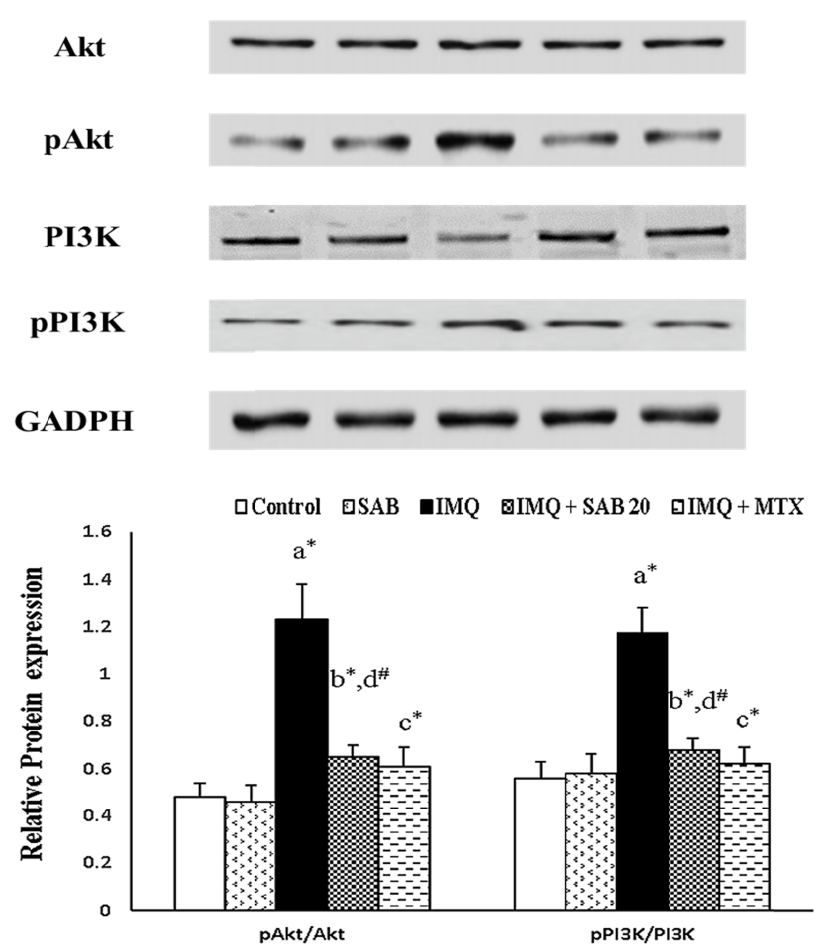

Fig. 3. Illustrate the protein expression of skin PI3K/Akt signaling molecules in control and IMQ-induced mice. Values are expressed as the mean \pm standard deviation. IMQ, imiquimod; $S A B$, salvianolic acid $B$; MTX, methotrexate; PI3K, phosphatidylinositol-3-kinase; Akt: protein kinase B. $p$-value $\left({ }^{*} p<0.05,{ }^{*} p<0.01\right)$ : where 'a' denotes the comparison between IMQ vs. Control, ' $b$ ' denotes the comparison between IMQ + $S A B$ vs. IMQ, 'C' denotes the comparison between IMQ + MTX vs. IMQ, 'd' denotes the comparison between IMQ + SAB vs. IMQ + MTX. 
A
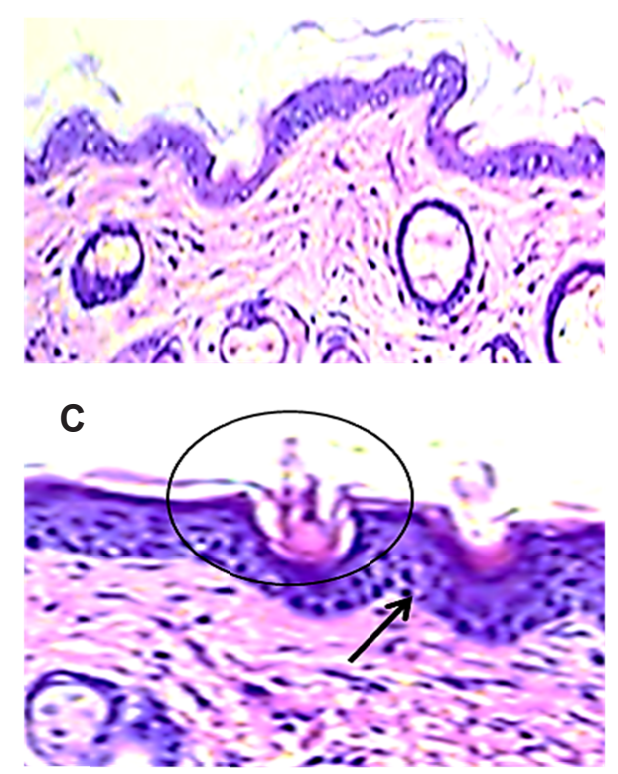

B
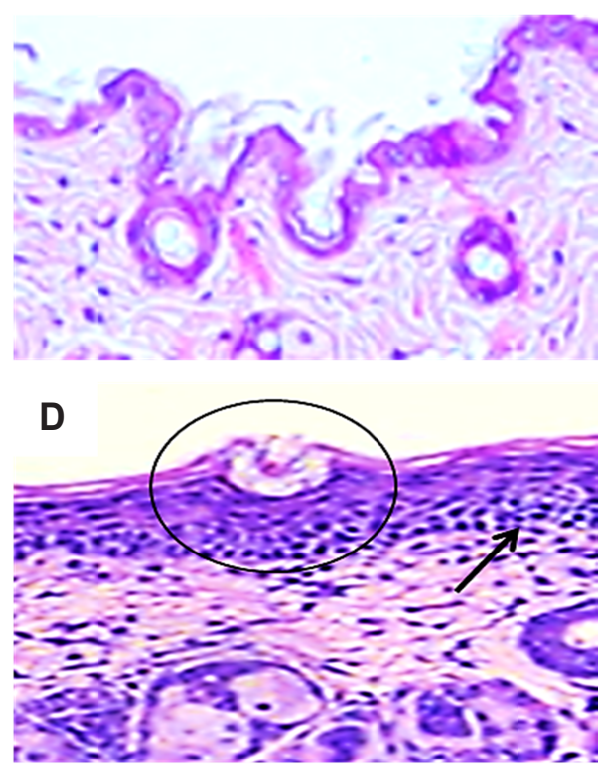

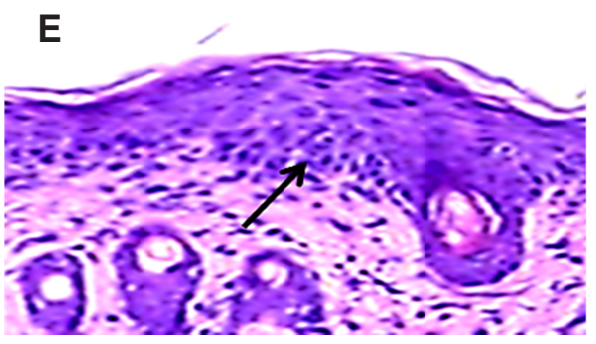

Fig. 4. Illustrates the histopathological changes in dermal tissue upon staining with H\&E (100x magnification) in control and IMQ-induced mice. Control (A) and SAB (B) mice dermal tissue slide display normal smoother epidermis without any inflammation or lesion. The IMQ induced mice dermal section (C) showed thickened epidermis (hyperplasia and acanthosis-circle) with elevated immune cell infiltration (arrow mark). Nevertheless, the mice dermal section treated with SAB (D) and MTX (E) portrait lesser thick epidermis (circle) with decreased immune cell infiltration (arrow mark) than IMQ-induced mice section. Scale bar: $100 \mu \mathrm{m}$. IMQ, imiquimod; SAB, salvianolic acid B.

upon staining with $\mathrm{H} \& \mathrm{E}$ in control and IMQ-induced mice. Control (Fig. 4A) and SAB (Fig. 4B) mice dermal tissue slide display normal smoother epidermis without any inflammation or lesion. The IMQ induced mice dermal section (Fig. 4C) showed thickened epidermis (hyperplasia and acanthosis) with elevated immune cell (neutrophil) infiltration. Nevertheless, the mice dermal section treated with SAB (Fig. 4D) and MTX (Fig. 4E) portrait lesser thick epidermis with decreased immune cell (neutrophil) infiltration than the IMQ-induced mice section. Overall, MTX standard drug-treated mice exhibit slightly better anti-psoriasis effect $(\mathrm{p}<0.05)$ than SAB treated mice in terms of PASI, skin thickness, antioxidant status, inflammatory markers as well as the protein expressions of keratin markers and PI3K/Akt signaling pathway.

\section{DISCUSSION}

Ample amount of studies indicated that topical application of IMQ (5\%) could trigger immune system via toll-like receptor 7/8 (TLR 7/8) which results in hyperstimulation of dendritic cells and hyperproliferation of keratinocytes (epidermis) and eventually upregulate various inflammatory cytokines (modulating IL-23/17 axis) and resembles human-plaque like psoriasis $[1,18]$. Therefore, the IMQ-exposed psoriasis-like mice model is a well-accepted psoriasis model (similar pathophysiology) to examining the anti-psoriasis activity of any drug [6]. Hence, the IMQ-induced psoriasis mouse model (BALB/c mice- highly susceptible to immune changes) was employed for this study to assess the antipsoriatic activity of $\mathrm{SAB}$ and compared with a standard druglike MTX (potent immunomodulatory/suppressive property). Before this study, we also conducted dose-dependent preliminary studies with SAB in IMQ-model to choose an effective dose, the result indicated that $40 \mathrm{mg} / \mathrm{kg}$ of SAB can effectively lower PASI 
and histological changes (data not shown). Therefore, the $40 \mathrm{mg} /$ $\mathrm{kg}$ of SAB was only used and compared with MTX. The outcome of this animal study clearly indicated that BALB/c mice treated with SAB or MTX significantly lowered the levels of PASI, skin thickness, lipid peroxidation product production (free radicals), inflammatory markers as well as modulating the protein expressions of keratin markers and PI3K/Akt signaling pathway.

The spleen index was determined to check the dynamic body changes as it is expressed based on spleen and body weight ratio. Mice topically applied with IMQ showed splenomegaly (enlarged spleen) through systemic effect and thus the spleen index was notably increased. Nevertheless, BALB/c mice SAB or MTX treatment significantly reduces the spleen index by reversing splenomegaly through decreasing cellularity (T cells) of periarteriolar lymphoid sheaths (PALS) and by lowering immune cell infiltration in the spleen. Previously, Elmore [20], also indicated the involvement of increased cellularity of PALS for splenomegaly in IMQ-induced psoriasis model. Aforementioned, IMQ induces hyperproliferation of dendritic cells and enhances keratinocyte proliferation which subsequently leads to scaling, redness/erythema as well as thickening of the epidermis (acanthosis) and increased inflammatory cell infiltration [18]. Likewise, during this study also we also encountered similar results with elevated psoriatic plaque, erythema, scaling with increases PASI, scaling, erythema scores along with increased skin thickening along with considerable psoriatic morphological changes (hyperplasia and acanthosis with elevated neutrophil infiltration) in IMQ-induced mice. While, 7 days of supplementation with SAB or MTX significantly suppressed those elevated PASI, scaling, erythema scores, skin thickness and psoriatic morphological changes (lesser hyperplasia with reduced neutrophil infiltration) due to its antiinflammatory, immunomodulatory and dermaprotective activities $[12,15]$.

A growing body of evidence indicates that excessive free radical production (reactive oxygen species) with abolished antioxidants (oxidative stress) is one of the major pathological events which contributes to psoriasis [21,22]. Hence, the antioxidant activity including CAT and SOD in dermal tissue as well as lipid peroxidation products (MDA) was determined for this study. The levels of lipid peroxidation products like MDA were elevated along with decreased antioxidants like SOD and CAT in IMQ applied mice due to excessive free radical generation. Nevertheless, the mice treated with SAB or MTX significantly decreased the production of free radicals and thus improve antioxidant activity with lowered lipid peroxidation product (MDA) formation due to potent antioxidant and free radical scavenging as well as anti-lipid peroxidative activities [14,23]. Moreover, Zhao and others [24], hinted that salvianolic acid B display potent free radical scavenging activity owing to its free hydroxyl group. For the present study, we plan to examine various inflammatory markers like IL-23, 22, 17A, $1 \beta$ and TNF- $\alpha$. Since those pro-inflammatory cytokines (markers) plays a pivotal role in the pathogenesis of psoriasis along with oxidative stress (as mentioned before). A pronounced increase in the concentration of various dermal inflammatory markers/ mediators (IL-23, 22, 17A, $1 \beta$ and TNF- $\alpha$ ) was observed in IMQinduced mice. But, those skin inflammatory markers/mediators (IL-23, 22, 17A, $1 \beta$, and TNF- $\alpha$ ) were significantly lowered upon supplementation with SAB or MTX in BALB/c mice. Previously, Zhang and Wang [25], also demonstrated that salvianolic acid B could significantly inhibit various inflammatory markers like IL$1 \beta, 6$, and TNF- $\alpha$ in human aortic smooth muscle cells because of its anti-inflammatory and antioxidant properties.

Many scientists have demonstrated that hyperproliferation of keratinocytes (epidermis) via upregulating various keratins especially $\mathrm{K} 16$ and $\mathrm{K} 17$ is recognized as a hallmark of psoriasis [26,27]. However, the expression of these keratins (K16 and K17) in normal keratinocytes were zero or minimal and are tightly regulated by various signaling pathway [28]. K16 is a type 1 cytokeratin, which strongly pairs with $\mathrm{K} 6$ and plays a major role in intermediate filament formation especially during the psoriatic condition. Whereas, $\mathrm{K} 17$ is also a type 1 cytokeratin (a cytoskeletal protein) and display a similar role as K16. Both K16 and 17 were found in the abnormal sebaceous gland, nail beds, and hair follicles and directly contribute to psoriasis as they both act as immunogenic antigens and trigger an immune response [29,30]. Hence, for the present study, the protein expression of both keratin K16 and K17 were examined using the western blot technique. The protein expression of both keratins (K16 and K17) were significantly upregulated in IMQ applied BALB/c mice due to immunogenic stimuli (IMQ) via TLR 7/8 by upregulating pro-inflammatory cytokines like IL-17 and 23 which results in upregulation of K16 and K17 and subsequently end up in hyperproliferation of keratinocytes. The above results are in corroboration with the results of Zhang and his co-workers [31], also confirmed that induction of IMQ would significantly upregulate K16 and K17. But mice treated with SAB or MTX showed considerable downregulation of these keratins (K16 and K17). The reason might be a potent anti-inflammatory and immune-suppressive activity of both SAB and MTX $[15,32]$.

Based on the above results it's clear that SAB could downregulate keratin expression and thus favor anti-hyperproliferation of keratinocytes, but the mechanism is still unknown. Moreover, numerous research groups hinted that the PI3K/Akt pathway inhibitors might prevent the psoriasis development in various psoriasis model particularly in the IMQ-induced mice model $[33,34]$. Therefore, the author hypothesizes that SAB would exert antihyperproliferation of keratinocytes (anti-psoriasis) by regulating PI3K/Akt signaling pathway. Topically application of 5\% IMQ on the back of mice showed greater upregulation of PI3K/Akt signaling molecules (pAkt/Akt and pPI3K/PI3K ratio). Likewise, Chamcheu et al. [35], showed that mice induced with IMQ showed enhanced protein expression of PI3K/Akt signaling molecules in the IMQ-induced mice model. Nonetheless, mice administered with SAB or MTX, significantly suppressed the activation of PI3K/ 
Akt signaling molecules by lowering phosphorylation of PI3K 110 and $\mathrm{Akt} \mathrm{Ser}^{473}$ (serine residue 473) which reflects in lowered pAkt/Akt and pPI3K/PI3K ratio. Salvianolic acid B was reported to inhibit oral squamous cell carcinoma by downregulating PI3K/ Akt signaling molecules (inactivate PI3K/Akt signaling pathway) and thus protect cells from hyperproliferation or hyperplasia [13]. Overall, SAB display potent anti-psoriasis activity by downregulating keratin markers (K16 and K17) through inactivating PI3K/ Akt signaling molecules. A few limitations of this study are the avoidance of measurement of the dermal concentration of $\mathrm{SAB}$ or MTX, as well as the expression of various immune cells and its proliferative property.

This animal study clearly demonstrated that BALB/c mice treated with SAB or MTX significantly lowered the levels of PASI, skin thickness, lipid peroxidation product production (free radicals), inflammatory markers and keratin markers through modulating PI3K/Akt signaling pathway and thus exhibit its strong anti-psoriatic property. However, clinical trials are needed to confirm the anti-psoriatic property of SAB before recommending to psoriasis patients.

\section{ACKNOWLEDGEMENTS}

All the authors are thankful to "Wuhan Hospital of Traditional Chinese Medicine (WH-2S4002) for financial support.

\section{CONFLICTS OF INTEREST}

The authors declare no conflicts of interest.

\section{REFERENCES}

1. Chen HH, Chao YH, Chen DY, Yang DH, Chung TW, Li YR, Lin CC. Oral administration of acarbose ameliorates imiquimodinduced psoriasis-like dermatitis in a mouse model. Int Immunopharmacol. 2016;33:70-82.

2. Mahajan R, Handa S. Pathophysiology of psoriasis. Indian J Dermatol Venereol Leprol. 2013;79 Suppl 7:S1-9.

3. Alharbi T, Alzahrani A, Hakami A, Almohammadi A, Alsaadi A, Magbel NB, AlJuaid N, Almasoudi H, Alruwaili T, Alkhezzi S. Psoriasis pathophysiology and impact on life. Int J Commun Med Public Health. 2018;5:3663-3667.

4. Conrad C, Gilliet M. Psoriasis: from pathogenesis to targeted therapies. Clin Rev Allergy Immunol. 2018;54:102-113.

5. Lowes MA, Bowcock AM, Krueger JG. Pathogenesis and therapy of psoriasis. Nature. 2007;445:866-873.

6. Li Y, Zhang G, Chen M, Tong M, Zhao M, Tang F, Xiao R, Wen H. Rutaecarpine inhibited imiquimod-induced psoriasis-like dermatitis via inhibiting the NF- $\mathrm{kB}$ and TLR7 pathways in mice. Biomed Pharmacother. 2019;109:1876-1883.
7. Krueger JG, Ferris LK, Menter A, Wagner F, White A, Visvanathan S, Lalovic B, Aslanyan S, Wang EE, Hall D, Solinger A, Padula S, Scholl P. Anti-IL-23A mAb BI 655066 for treatment of moderate-tosevere psoriasis: Safety, efficacy, pharmacokinetics, and biomarker results of a single-rising-dose, randomized, double-blind, placebocontrolled trial. J Allergy Clin Immunol. 2015;136:116-124.e7.

8. Cline A, Hill D, Lewallen R, Feldman SR. Current status and future prospects for biologic treatments of psoriasis. Exp Review Clin Immunol. 2016;12:1273-1287.

9. Talbott W, Duffy N. Complementary and alternative medicine for psoriasis: what the dermatologist needs to know. Am J Clin Dermatol. 2015;16:147-165.

10. Farahnik B, Sharma D, Alban J, Sivamani RK. Topical botanical agents for the treatment of psoriasis: a systematic review. Am J Clin Dermatol. 2017;18:451-468

11. Liu QS, Luo XY, Jiang H, Xing Y, Yang MH, Yuan GH, Tang Z, Wang H. Salvia miltiorrhiza injection restores apoptosis of fibroblast-like synoviocytes cultured with serum from patients with rheumatoid arthritis. Mol Med Rep. 2015;11:1476-1482.

12. Fan Y, Luo Q, Wei J, Lin R, Lin L, Li Y, Chen Z, Lin W, Chen Q. Mechanism of salvianolic acid B neuroprotection against ischemia/ reperfusion induced cerebral injury. Brain Res. 2018;1679:125-133.

13. Wei J, Wu J, Xu W, Nie H, Zhou R, Wang R, Liu Y, Tang G, Wu J. Salvianolic acid B inhibits glycolysis in oral squamous cell carcinoma via targeting PI3K/AKT/HIF-1a signaling pathway. Cell Death Dis. 2018;9:599.

14. Ma ZG, Xia HQ, Cui SL, Yu J. Attenuation of renal ischemic reperfusion injury by salvianolic acid B via suppressing oxidative stress and inflammation through PI3K/Akt signaling pathway. Braz J Med Biol Res. 2017;50:e5954.

15. Xia ZB, Yuan YJ, Zhang QH, Li H, Dai JL, Min JK. Salvianolic Acid B Suppresses Inflammatory Mediator Levels by Downregulating NF- $\kappa B$ in a Rat Model of Rheumatoid Arthritis. Med Sci Monit. 2018;24:2524-2532.

16. Chen YS, Lee SM, Lin YJ, Chiang SH, Lin CC. Effects of Danshensu and Salvianolic Acid B from Salvia miltiorrhiza Bunge (Lamiaceae) on cell proliferation and collagen and melanin production. Molecules. 2014;19:2029-2041.

17. El Malki K, Karbach SH, Huppert J, Zayoud M, Reissig S, Schüler R, Nikolaev A, Karram K, Münzel T, Kuhlmann CR, Luhmann HJ, von Stebut E, Wörtge S, Kurschus FC, Waisman A. An alternative pathway of imiquimod-induced psoriasis-like skin inflammation in the absence of interleukin-17 receptor a signaling. J Invest Dermatol. 2013;133:441-451.

18. Zhang S, Liu X, Mei L, Wang H, Fang F. Epigallocatechin-3-gallate (EGCG) inhibits imiquimod-induced psoriasis-like inflammation of BALB/c mice. BMC Complement Altern Med. 2016;16:334.

19. Zhang Q, Hu LQ, Li HQ, Wu J, Bian NN, Yan G. Beneficial effects of andrographolide in a rat model of autoimmune myocarditis and its effects on PI3K/Akt pathway. Korean J Physiol Pharmacol. 2019;23:103-111.

20. Elmore SA. Enhanced histopathology of the spleen. Toxicol Pathol. 2006;34:648-655.

21. Lin X, Huang T. Oxidative stress in psoriasis and potential therapeutic use of antioxidants. Free Radic Res. 2016;50:585-595.

22. Zhou Q, Mrowietz U, Rostami-Yazdi M. Oxidative stress in the pathogenesis of psoriasis. Free Radic Biol Med. 20091;47:891-905. 
23. Jiang YF, Liu ZQ, Cui W, Zhang WT, Gong JP, Wang XM, Zhang Y, Yang MJ. Antioxidant effect of salvianolic acid B on hippocampal CA1 neurons in mice with cerebral ischemia and reperfusion injury. Chin J Integr Med. 2015;21:516-522.

24. Zhao GR, Zhang HM, Ye TX, Xiang ZJ, Yuan YJ, Guo ZX, Zhao LB. Characterization of the radical scavenging and antioxidant activities of danshensu and salvianolic acid B. Food Chem Toxicol. 2008;46:73-81.

25. Zhang HS, Wang SQ. Salvianolic acid B from Salvia miltiorrhiza inhibits tumor necrosis factor-alpha (TNF-alpha)-induced MMP-2 upregulation in human aortic smooth muscle cells via suppression of $\mathrm{NAD}(\mathrm{P}) \mathrm{H}$ oxidase-derived reactive oxygen species. J Mol Cell Cardiol. 2006;41:138-148.

26. Jin L, Wang G. Keratin 17: a critical player in the pathogenesis of psoriasis. Med Res Rev. 2014;34:438-454.

27. Leigh IM, Navsaria H, Purkis PE, McKay IA, Bowden PE, Riddle PN. Keratins (K16 and K17) as markers of keratinocyte hyperproliferation in psoriasis in vivo and in vitro. Br J Dermatol. 1995;133:501511.

28. Zhang W, Dang E, Shi X, Jin L, Feng Z, Hu L, Wu Y, Wang G. The pro-inflammatory cytokine IL-22 up-regulates keratin 17 expression in keratinocytes via STAT3 and ERK1/2. PLoS One. 2012;7:e40797.

29. Yang L, Fan X, Cui T, Dang E, Wang G. Nrf2 promotes keratinocyte proliferation in psoriasis through up-regulation of keratin 6, keratin
16, and keratin 17. J Invest Dermatol. 2017;137:2168-2176.

30. Du ZC, Xue T, Jiang M, Lu HY, Ye ZC, Ruan BJ, Xu CM, Jiang YH, Wei M, Wang G, Lu ZF, Lei XY, Wang L. Arctigenin attenuates imiquimod-induced psoriasis-like skin lesions via down-regulating keratin17. Int J Clin Exp Med. 2016;9:1639-1647.

31. Zhang J, Li X, Wei J, Chen H, Lu Y, Li L, Han L, Lu C. Gallic acid inhibits the expression of keratin 16 and keratin 17 through Nrf2 in psoriasis-like skin disease. Int Immunopharmacol. 2018;65:84-95.

32. Dogra S, Mahajan R. Systemic methotrexate therapy for psoriasis: past, present and future. Clin Exp Dermatol. 2013;38:573-588.

33. Zhang M, Zhang X. The role of PI3K/AKT/FOXO signaling in psoriasis. Arch Dermatol Res. 2019;311:83-91.

34. Chamcheu JC, Adhami VM, Esnault S, Sechi M, Siddiqui IA, Satyshur KA, Syed DN, Dodwad SM, Chaves-Rodriquez MI, Longley BJ, Wood GS, Mukhtar H. Dual inhibition of PI3K/Akt and mTOR by the dietary antioxidant, delphinidin, ameliorates psoriatic features in vitro and in an imiquimod-induced psoriasis-like disease in mice. Antioxid Redox Signal. 2017;26:49-69.

35. Chamcheu JC, Chaves-Rodriquez MI, Adhami VM, Siddiqui IA, Wood GS, Longley BJ, Mukhtar H. Upregulation of PI3K/AKT/ mTOR, FABP5 and PPAR $\beta / \delta$ in human psoriasis and imiquimodinduced murine psoriasiform dermatitis model. Acta Derm Venereol. 2016;96:854-856. 